Published in final edited form as:

Curr Opin Rheumatol. 2019 May ; 31(3): 256-263. doi:10.1097/BOR.0000000000000591.

\title{
Patient Preferences for Rheumatoid Arthritis Treatment
}

\author{
Betty Hsiao, MD ${ }^{1}$ and Liana Fraenkel, MD, MPH ${ }^{1,2}$ \\ ${ }^{1}$ Yale University School of Medicine, New Haven, CT, 06520 \\ ${ }^{2}$ VA Connecticut Healthcare System, West Haven, CT, 06516
}

\begin{abstract}
1. Purpose of review: To provide an overview of recent articles discussing patient preferences for rheumatoid arthritis treatment.
\end{abstract}

2. Recent findings: Recent studies examined patient preferences for rheumatoid arthritis (RA) treatment in several populations, finding that most participants were willing to accept certain risks of adverse effects to gain potential benefits. Perspectives regarding cannabis were studied, with patients describing medical marijuana as an alternative therapy to be used with prescription medications or as means of tapering off these medications. Treatment preferences for different RA therapies were explored using a conjoint analysis survey and five distinct preference phenotypes emerged, with members of the largest group most concerned with the cost of medications. Other discrete choice studies demonstrated route of administration as an important attribute influencing treatment preferences, with patients expressing preference for various modes in different studies. Patient preferences for route of administration have demonstrated preference for newer autoinjectors over pre-filled syringes as well as currently marketed auto-injectors. Incorporating patient preferences in clinical practice recommendations was described in the development of the 2015 American College of Rheumatology (ACR) RA treatment guidelines as well as the 2017 ACR/ American Association of Hip and Knee Surgeons guidelines for perioperative management of antirheumatic medications. Additionally, other studies explored preferences with regard to predictive testing, medication intensification and tapering, treatment goals and psychological support.

3. Summary: Our review of recent studies show variability in patient preferences for RA treatment, highlighting the importance of incorporating patient input into the treatment approach.

\section{Keywords}

rheumatoid arthritis; patient preferences; treatment

\section{Introduction}

Current American College of Rheumatology (ACR) guidelines recommend a treat-to-target (T2T) approach for rheumatoid arthritis (RA) with the goal of attaining low-disease activity

Corresponding Author: Betty Hsiao, Yale University School of Medicine, Section of Rheumatology, 300 Cedar St, TAC Bldg, RM \#525, P.O. Box 208031, New Haven, CT, 06520-8031, tel 203 785 2453, fax 203785 7053, betty.hsiao@ yale.edu.

Conflicts of interest

The authors have no conflicts of interest. 
or clinical remission. (1) While routine monitoring and prompt escalation is recommended for the majority of patients who are not at target, guidelines do not endorse one intervention over another and treatment decisions should be based on using a shared decision-making approach. (2) In this review, we discuss recent manuscripts published from 2016-2018 that examine RA patient preferences for the prevention of RA, conventional and complementary treatments, medication intensification and reduction, treatment goals, and clinical practice recommendations.

\section{Preferences for Prevention of RA}

Finckh et al (3) used stated-preference surveys to examine risk thresholds for prevention among asymptomatic individuals at risk for RA (i.e. those with first-degree relatives with RA). Results showed only $7 \%$ of participants chose to take a preventive medication if the risk of developing RA was $1 \%$, compared to $30 \%$ and $38 \%$ if the risks were 20 and $40 \%$ respectively. Additionally, a reduction in the risk of developing RA by $20 \%$ or more and a lowered risk of developing serious adverse events (AEs) of $10 \%$ or less, were both significantly associated with the odds of initiating treatment.

\section{Preferences Regarding Disease-modifying Anti-rheumatic Drugs (DMARDs), Biologics and Small Molecule Drugs}

Treatment preferences of patients with early RA (less than two years since diagnosis) were assessed using a discrete-choice experiment. Participants weighed the chance of improving a major symptom (pain, swollen and tender joints) and reducing the chance of serious joint damage as most important. Patients considered a small risk of serious infections or a possible increased risk of cancer to be the most significant AEs but were willing to accept these risks for a $15 \%$ absolute increase in the chance of a major symptom improvement. Through latent-class analysis, two patient groups were identified: $54 \%$ were more risk averse (to increased cancer and/or infection risk) and the remaining focused almost exclusively on treatment benefits. (4)

Husni et al (5) evaluated preferences of patients with moderate-to-severe RA using a discrete choice experiment that included hypothetical RA treatments with varying levels of efficacy, AEs, and other attributes such as route of administration, dose frequency and out-of-pocket costs. Participants were willing to accept an increased risk of AEs (abnormal laboratory results, serious infections, and cancer) to achieve reductions in RA-related pain, number of swollen joints, and improvement in physical function. While both studies utilized discrete choice experiments to evaluate trade-offs in RA patient preferences, Husni et al conducted the first study in the United States to assess the thresholds of benefit-risk, which focused on the risk of cancer and serious infection.

Hazlewood et al $(6,7)$ sought to incorporate patient preferences into treatment recommendations, which is a key element in the standard, the Grading of Recommendations Assessment, Development, and Evaluation (GRADE) approach, for developing treatment recommendations. After completing a Cochrane network meta-analysis of methotrexate and methotrexate-based combination therapy with traditional and biologic DMARDs, they found that the development and grading the strength of treatment recommendation required 
balancing trade-offs between efficacy, potential side-effects, and dose regimen. The authors aimed to develop and demonstrate a novel Bayesian approach for combining the data of measured patients' preferences for these trade-offs from their previous study (4) with outcome estimates from the network meta-analysis for consideration of two clinical questions: 1) the choice of methotrexate versus triple therapy as initial treatment 2) the choice of triple therapy versus methotrexate and anti-tumor necrosis factor therapy in patients with an inadequate response to methotrexate. The authors found that 1) most patients (including methotrexate naïve patients) preferred triple therapy as the initial treatment, with a higher probability of symptom improvement outweighing additional pill burden and regular eye examinations, 2) patients with inadequate responses to methotrexate had varied preferences due to an increased number of trade-offs involving differences in outcomes, dosing regimens, rare AEs, and monitoring. $(6,7)$

Treatment preferences of RA patients for triple therapy, biologics, and Janus kinase inhibitors were measured by Fraenkel et al (8) using a conjoint analysis survey. Preferences were estimated based on patients' trade-offs across route of administration, onset of action, bothersome side effects, serious infections, very rare side effects, time on the market and affordability. Using latent class analysis, five preference phenotypes emerged: members of the largest group (38.4\%) were affected most by the cost of medications, members of the second largest group (25.8\%) were concerned about the risk of bothersome side effects, members of the third largest group (18\%) were most impacted by onset of action and risk of serious infections. The two smallest groups were most concerned about the risk of very rare side effects $(11.2 \%)$ and the route of administration (6.6\%).

Patient knowledge and perception of biosimilars were evaluated in 121 RA patients in Belgium (treated with both traditional synthetic DMARDs and/or biologics, but not biosimilars) through anonymous web surveys. The majority (79\%) of patients knew about biologics but only $49 \%$ had heard of biosimilars. The participants were asked to indicate "what they would wonder about" if a physician prescribed them an originator or biosimilar. Participants had similar concerns regarding the efficacy, side-effects and suitability of biosimilars and originators, but biosimilars were questioned more often in terms of their safety compared to originators. (9) Table 1 summarizes these findings.

\section{Preferences for DMARD Route of Administration}

Bolge et al (10) found over 50\% of RA patients (who were on DMARDs and had discussed but never taken biologic therapy) were open to both intravenous (IV) and subcutaneous (SQ) modes of administration, and that only $26.3 \%$ of patients strongly preferred SQ injection through self-administered online surveys. Nolla et al (11) utilized assessed values assigned to attributes of biologic therapies using conjoint analysis among treatment-experienced Spanish RA patients (diagnosed for at least two years prior to study and received biologics for at least one year) as well as rheumatologists. The relative importance of attributes was calculated and participants identified the ideal attributes for biologic therapies should allow relief from pain and functional capacity improvement, with a low risk of AEs, longer time period prior to perceiving the need for another dose, and self-administration at home. Additionally, patients placed more importance on the route of administration and a lower 
frequency of administration than rheumatologists, while providers placed higher importance on relief from pain, improvement in functional capacity, and risk of AEs. Louder et al (12) and Alten et al (13) both found that patients considered route of administration to be the most important attribute influencing treatment preferences in studies using choice-based conjoint surveys and discrete choice surveys respectively, with the majority of patients preferring the oral route of administration over other routes. Emadi et al (14) evaluated 294 RA patients using a questionnaire to determine their preferred route of administration of their RA treatment. Participants expressed the highest preference for oral therapy (69\%) as compared to injection (23\%) and IV (8\%) therapy; additionally, $85 \%$ of patients wished to remain on oral therapy as compared with $63 \%$ of patients on IV and $58 \%$ of patients on SQ therapies.

Bolge et al (15) conducted semi-structured telephone interviews with 405 patients receiving IV therapy [including 204 (50.4\%) with RA] and found the majority of patients preferred IV medication to SQ injection, with the most common reasons for preference for IV therapy were aversion to self-inject, less frequent dosing, and preference for administration by a health care professional. Gaylis et al (16) performed a single-center, non-interventional patient questionnaire-based study of 100 patients with inflammatory arthritis currently receiving IV biologics, including 31 patients who were previously treated with SQ medications, and similarly found patients had a favorable perception of IV therapy, with $90 \%$ patients rating their satisfaction with current IV therapy as 4 or 5 on a 5-point Likert scale. Desplats et al (17) analyzed preferences for switching from IV to SQ in 127 patients treated by tocilizumab and 74 patients by abatacept. Patients with favorable attitudes towards switching cited convenience (i.e., concerns about repeated hospital visits for IV infusions, greater autonomy with SQ injections, economic considerations), while those who rejected switching were concerned about lack of follow-up, absence of medical assistance during the SQ injection, fear of AEs, and fear of SQ injections.

Several studies evaluated patient preferences regarding the auto-injector mode of administration. The GO-SAVE study found that the majority of patients reported less discomfort, redness, pain, stinging, and burning with the golimumab auto-injector as compared with their prior adalimumab or etanercept injections. (18) The phase 3 SARILRA-EASY study assessed the use of auto-injector (pen) among RA patients administering sarilumab as compared to the prefilled syringe. In this study, the majority of patients reported ease of use and satisfaction with the auto-injector, with similar safety and efficacy as compared to the syringe. (19) The Home Use Study and Patient Preference Study evaluated the investigational AutoTouch ${ }^{\mathrm{TM}}$ reusable auto-injector versus the currently marketed single-use SureClick® auto-injector among patients with inflammatory arthritis, including RA. They found that more patients preferred the AutoTouch ${ }^{\mathrm{TM}}$ because of the added ease of self-injecting, pressing the start button, following injection process, and certainty of knowing when the injection was completed. (20) Egeth et al (21) found that RA patients preferred the Brenzys auto-injector, which does not have a push button requiring the thumb and is administered in fewer steps, over the Enbrel auto-injector. (21) Table 2 summarizes these findings. 


\section{Preferences for Glucocorticoids}

Black et al (22) evaluated patient perspectives of glucocorticoids with a cross-sectional survey in patients with rheumatic diseases, including $25 \%$ with RA. More than half of the participants felt that glucocorticoids significantly improved their symptoms and the benefits were greater than the AEs. Patients rated skin thinning and/or easy bruisability, sleep disturbance, mood disturbance, and change in facial shape as the worst AEs.

\section{Preferences for Cannabis}

While there was a lack of recent studies exploring patient preferences for complementary or alternative treatments, Bruce et al (23) studied the utilization of medical cannabis among patients with chronic conditions such as RA through semi-structured telephone interviews. Participants described using medical marijuana as an alternative to other medications (most commonly opioids, but also anticonvulsants, anti-inflammatories, and over-the-counter analgesics), as a treatment to be used along with prescription medications, and as a way to help them taper off prescription medications. Patients reported concerns regarding the toxicity, dependence and tolerance of all prescription medications, particularly opioids. They reported that medical cannabis improves management of certain symptoms such as pain, has a quicker onset of action and longer lasting effects than prescription drugs.

\section{Preferences Related to Clinical Practice Recommendations}

Fraenkel et al (24) evaluated how to best incorporate patient preferences in the development of RA treatment recommendations. In a pilot study conducted in conjunction with the ACR's development of the 2015 RA Recommendations, they sought to determine the feasibility and value of developing clinical practice guidelines based on a voting panel comprised of patients. Patient recommendations were then compared with those developed by the physician-dominated voting panel. For 13 of the 16 questions, the patient panel recommended the same course of action as did the physician-dominated panel; however, the two panels viewed trade-offs between benefits and harms differently for three of the questions. For two of the questions, patients voted conditionally for using triple therapy (versus mono DMARD) for DMARD-naive patients with early and established RA with at least moderate disease activity versus the physician-dominated panel who voted conditionally against triple therapy. Patients felt that the increased chance of significant improvement and the lack of significant added toxicity justified the use of all three medications. Additionally, the two panels also differed in their recommendations for using tofacitinib: the patient panel voted for using tofacitinib over methotrexate in DMARD-naïve RA patients (because of the statistically significant benefits associated with tofacitinib and its lower risk of gastrointestinal side effects as compared to methotrexate), whereas the physician-dominated panel voted against using tofacitinib in this population. Goodman et al (25) described how input from a patient panel influenced the development of the 2017 ACR/ American Association of Hip and Knee Surgeons clinical practice guidelines for perioperative management of anti-rheumatic medications in patients undergoing elective total hip or total knee arthroplasty. The patient panel consisted of $11 \mathrm{RA}$ or juvenile idiopathic arthritis patients all with prior personal experience of joint replacements who voted anonymously on the drafted recommendations until an $80 \%$ or higher consensus was 
reached. The patient panel placed higher importance on avoiding an infection than a disease flare, citing that flares represented a "known risk" that could potentially be controlled and treated, in contrast to infections, which were perceived to have the risk of much worse outcomes (e.g. permanent loss of prosthesis, amputation, prolonged hospitalization, disability and death).

\section{Preferences for Predictive Testing}

Patient perceptions on the value of predictive testing for treatments was studied by Kumar et al (26) in a group of patients with established RA, (range of disease duration from three to 34 years), using qualitative interviews and found that overall, patients supported the development of predictive testing to predict response to treatments. Patients also expressed the need for explanations that described the consequences of untreated RA and delayed treatment response as well as support to balance the trade-offs between the risks of testing that may be invasive and/or have varying accuracies with the potential benefits of new information to help identify a suitable treatment.

\section{Preferences for Medication Intensification}

Patient preferences regarding medication intensification were evaluated in patients with established RA, with a median disease duration of eight years using questionnaires regarding perceived health change, satisfaction with current health, willingness to change therapy, and expected health change until the next visit. Hendrikx et al (27) used logistic regression to assess independent associations between these measures, clinical measures and actual DMARD/biologic intensification, finding that all patient perception measures exhibited significant associations independent of clinical measures, with patients' willingness to escalate therapy strongly associated with their satisfaction with their current health state.

\section{Preferences for Medication Dose Reduction}

Patient preferences for dose reduction were evaluated by Verhoef et al (28) using a mixed methods study that identified the factors that play a role for RA patients when considering a gradual tapering of their biologic until discontinuation. Most participants reported a positive attitude toward biologic dose reduction but expressed concerns about a possible disease flare that may cause increased pain and/or loss of function. Additionally, patients expressed the importance for them to know the possibility to increase the dose if the plan for dose reduction is unsuccessful.

\section{Preferences for Treatment Goals}

Patient preferences for treatment goals were investigated in a qualitative study among young patients with inflammatory arthritis. Hart et al (29) found that these patients valued treatment that helps them achieve living a "normal life", i.e. a reference to their prediagnosis states or to others. They also perceived treatment as both an opportunity to achieving relief from symptoms as well as a threat to achieving a normal life through experiencing side effects. These young patients discussed having strong emotions about aspects of treatment, acquiring information from different sources, and acknowledged the uncertainty associated with new treatments. 


\section{Preferences for Psychological Support}

Dures et al (30) evaluated preferences for psychological support in patients with inflammatory arthritis in a multi-center study. While only $23 \%$ of patients reported routinely being asked about social and emotional issues by rheumatology professionals, $46 \%$ wanted the opportunity to discuss psychological impact. Additionally, patients expressed the desire for support managing the impact of pain and fatigue (82\%), managing emotions (57\%), work and leisure (52\%), relationships (37\%), and depression (34\%). Only 6\% of patients stated that social and emotional issues were irrelevant. Dwarswaard et al (31) performed a qualitative review to evaluate self-management support from the perspective of patients with chronic conditions including RA. They reviewed 37 articles and found patients with chronic disease need instrumental, psychosocial, and relational support from different sources, including health-care professionals.

\section{Conclusion}

This review summarizes recent publications that discuss patient preferences across different aspects of RA treatment, highlighting the variability of patient preferences and thus the importance of adopting a treatment approach based on mutual patient-clinician consideration of patient input using shared decision-making.

\section{Acknowledgments}

Financial support and sponsorship

\section{References}

1. Singh JA, Saag KG, Bridges SL, Jr., et al. 2015 American College of Rheumatology Guideline for the Treatment of Rheumatoid Arthritis. Arthritis care \& research. 2016;68(1):1-25. [PubMed: 26545825]

2. Elwyn G, Frosch D, Thomson R, et al. Shared decision making: a model for clinical practice. Journal of general internal medicine. 2012;27(10):1361-7. [PubMed: 22618581]

3. Finckh A, Escher M, Liang MH, Bansback N. Preventive Treatments for Rheumatoid Arthritis: Issues Regarding Patient Preferences. Curr Rheumatol Rep. 2016;18(8):51. [PubMed: 27402108]

*4. Hazlewood GS, Bombardier C, Tomlinson G, et al. Treatment preferences of patients with early rheumatoid arthritis: a discrete-choice experiment. Rheumatology (Oxford). 2016;55(11):1959_ 68. [PubMed: 27477807]

*5. Husni ME, Betts KA, Griffith J, et al. Benefit-risk trade-offs for treatment decisions in moderateto-severe rheumatoid arthritis: focus on the patient perspective. Rheumatol Int. 2017;37(9):142334. [PubMed: 28623526]

6. Hazlewood GS. Measuring Patient Preferences: An Overview of Methods with a Focus on Discrete Choice Experiments. Rheum Dis Clin North Am. 2018;44(2):337-47. [PubMed: 29622300]

7. Hazlewood GS, Bombardier C, Tomlinson G, Marshall D. A Bayesian model that jointly considers comparative effectiveness research and patients' preferences may help inform GRADE recommendations: an application to rheumatoid arthritis treatment recommendations. J Clin Epidemiol. 2018;93:56-65. [PubMed: 29051108]

**8. Fraenkel L, Nowell WB, Michel G, Wiedmeyer C. Preference phenotypes to facilitate shared decision-making in rheumatoid arthritis. Ann Rheum Dis. 2018;77(5):678-83. [PubMed: 29247126] 
9. van Overbeeke E, De Beleyr B, de Hoon J, et al. Perception of Originator Biologics and Biosimilars: A Survey Among Belgian Rheumatoid Arthritis Patients and Rheumatologists. BioDrugs. 2017;31(5):447-59. [PubMed: 28929342]

10. Bolge SC, Goren A, Brown D, et al. Openness to and preference for attributes of biologic therapy prior to initiation among patients with rheumatoid arthritis: patient and rheumatologist perspectives and implications for decision making. Patient Prefer Adherence. 2016;10:1079-90. [PubMed: 27390518]

11. Nolla JM, Rodriguez M, Martin-Mola E, et al. Patients' and rheumatologists' preferences for the attributes of biological agents used in the treatment of rheumatic diseases in Spain. Patient Prefer Adherence. 2016;10:1101-13. [PubMed: 27382258]

12. Louder AM, Singh A, Saverno K, et al. Patient Preferences Regarding Rheumatoid Arthritis Therapies: A Conjoint Analysis. Am Health Drug Benefits. 2016;9(2):84-93.

13. Alten R, Kruger K, Rellecke J, et al. Examining patient preferences in the treatment of rheumatoid arthritis using a discrete-choice approach. Patient Prefer Adherence. 2016;10:2217-28. [PubMed: 27843301]

14. Emadi SA, Hammoudeh M, Mounir M, et al. An assessment of the current treatment landscape for rheumatology patients in Qatar: Recognising unmet needs and moving towards solutions. J Int Med Res. 2017;45(2):733-43. [PubMed: 28415924]

15. Bolge SC, Eldridge HM, Lofland JH, et al. Patient experience with intravenous biologic therapies for ankylosing spondylitis, Crohn's disease, psoriatic arthritis, psoriasis, rheumatoid arthritis, and ulcerative colitis. Patient Prefer Adherence. 2017;11:661-9. [PubMed: 28405158]

16. Gaylis NB, Sagliani J, Black S, et al. Patient-reported outcome assessment of inflammatory arthritis patient experience with intravenously administered biologic therapy. Patient Prefer Adherence. 2017;11:1543-53. [PubMed: 28979103]

17. Desplats M, Pascart T, Jelin G, et al. Are abatacept and tocilizumab intravenous users willing to switch for the subcutaneous route of administration? A questionnaire-based study. Clin Rheumatol. 2017;36(6):1395-400. [PubMed: 28255739]

18. Dehoratius RJ, Brent LH, Curtis JR, et al. Satisfaction with Subcutaneous Golimumab and its Auto-Injector among Rheumatoid Arthritis Patients with Inadequate Response to Adalimumab or Etanercept. Patient. 2018;11(3):361-9. [PubMed: 29427176]

19. Kivitz A, Baret-Cormel L, van Hoogstraten H, et al. Usability and Patient Preference Phase 3 Study of the Sarilumab Pen in Patients with Active Moderate-to-Severe Rheumatoid Arthritis. Rheumatol Ther. 2018;5(1):231-42. [PubMed: 29209946]

20. Collier DH, Bitman B, Coles A, et al. A novel electromechanical autoinjector, AutoTouch, for selfinjection of etanercept: real-world use and benefits. Postgrad Med. 2017;129(1):118-25. [PubMed: 27829325]

21. Egeth M, Soosaar J, Nash P, et al. Patient and Healthcare Professionals Preference for Brenzys vs. Enbrel Autoinjector for Rheumatoid Arthritis: A Randomized Crossover Simulated-Use Study. Adv Ther. 2017;34(5):1157-72. [PubMed: 28417318]

22. Black RJ, Goodman SM, Ruediger C, et al. A Survey of Glucocorticoid Adverse Effects and Benefits in Rheumatic Diseases: The Patient Perspective. J Clin Rheumatol. 2017;23(8):416-20. [PubMed: 28926469]

23. Bruce D, Brady JP, Foster E, Shattell M. Preferences for Medical Marijuana over Prescription Medications Among Persons Living with Chronic Conditions: Alternative, Complementary, and Tapering Uses. J Altern Complement Med. 2018;24(2):146-53. [PubMed: 28945457]

**24. Fraenkel L, Miller AS, Clayton K, et al. When Patients Write the Guidelines: Patient Panel Recommendations for the Treatment of Rheumatoid Arthritis. Arthritis Care Res (Hoboken). 2016;68(1):26-35. [PubMed: 26545701]

**25. Goodman SM, Miller AS, Turgunbaev M, et al. Clinical Practice Guidelines: Incorporating Input From a Patient Panel. Arthritis Care Res (Hoboken). 2017;69(8):1125-30. [PubMed: 28620968]

26. Kumar K, Peters S, Barton A, Matura. Rheumatoid arthritis patient perceptions on the value of predictive testing for treatments: a qualitative study. BMC Musculoskelet Disord. 2016;17(1):460. [PubMed: 27825322] 
*27. Hendrikx J, Kievit W, Fransen J, van Riel PL. The influence of patient perceptions of disease on medication intensification in daily practice. Rheumatology (Oxford). 2016;55(11):1938-45. [PubMed: 27009827]

28. Verhoef LM, Selten EMH, Vriezekolk JE, et al. The patient perspective on biologic DMARD dose reduction in rheumatoid arthritis: a mixed methods study. Rheumatology (Oxford). 2018;57(11): 1947-55. [PubMed: 30010899]

29. Hart RI, McDonagh JE, Thompson B, et al. Being as Normal as Possible: How Young People Ages 16-25 Years Evaluate the Risks and Benefits of Treatment for Inflammatory Arthritis. Arthritis Care Res (Hoboken). 2016;68(9):1288-94. [PubMed: 27040737]

30. Dures E, Almeida C, Caesley J, et al. Patient preferences for psychological support in inflammatory arthritis: a multicentre survey. Ann Rheum Dis. 2016;75(1):142-7. [PubMed: 25261572]

31. Dwarswaard J, Bakker EJ, van Staa A, Boeije HR. Self-management support from the perspective of patients with a chronic condition: a thematic synthesis of qualitative studies. Health Expect. 2016;19(2):194-208. [PubMed: 25619975] 


\section{Key Points:}

- Recent studies show variability in patient preferences across different aspects of RA treatment, highlighting the importance of implementing shareddecision making in the treatment approach.

- $\quad$ Most patients expressed willingness to accept certain risks of adverse effects to potentially gain benefits of treatment.

- The development of the 2015 ACR RA treatment guidelines and 2017 ACR/ American Association of Hip and Knee Surgeons guidelines for perioperative management of anti-rheumatic medications both utilized the Grading of Recommendations Assessment, Development, and Evaluation methodology, which acknowledges the critical role of patient input in treatment recommendations. 


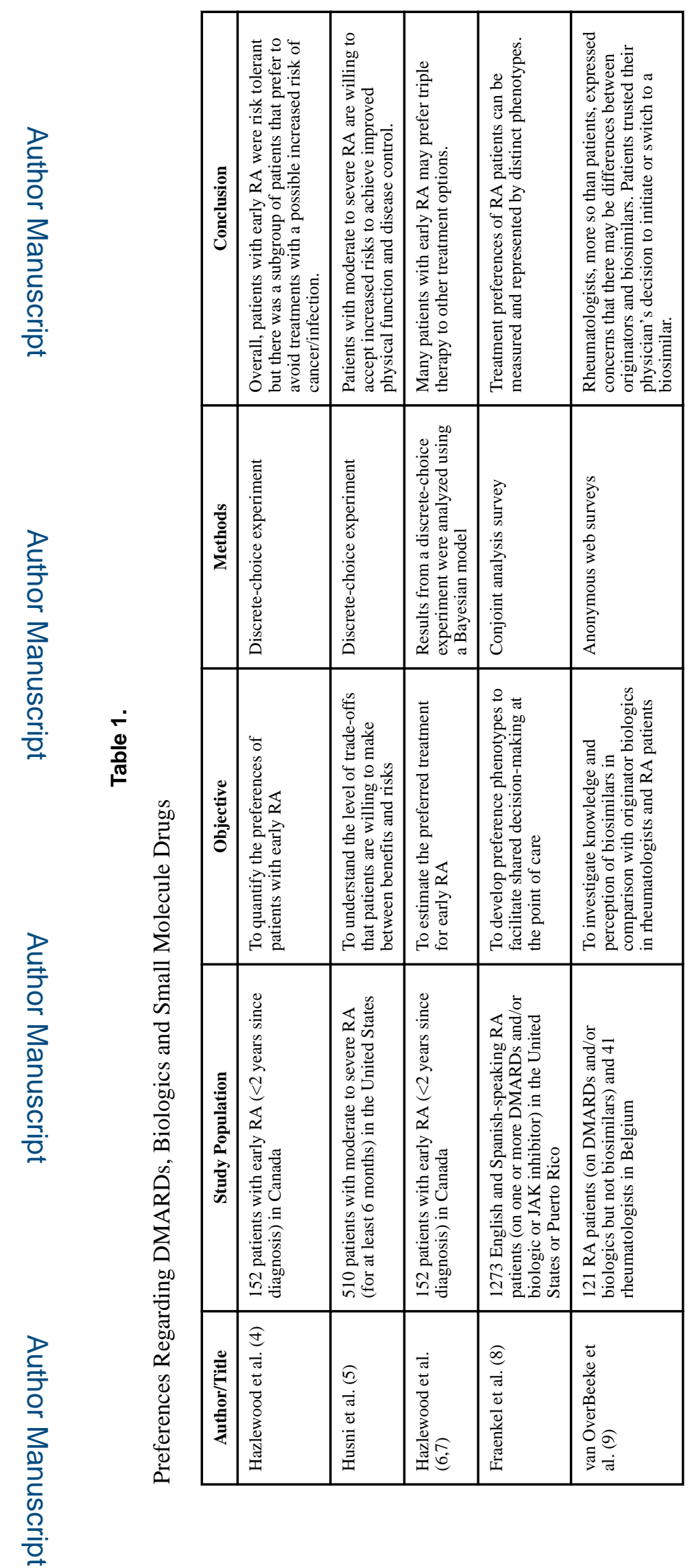

Curr Opin Rheumatol. Author manuscript; available in PMC 2020 May 01. 


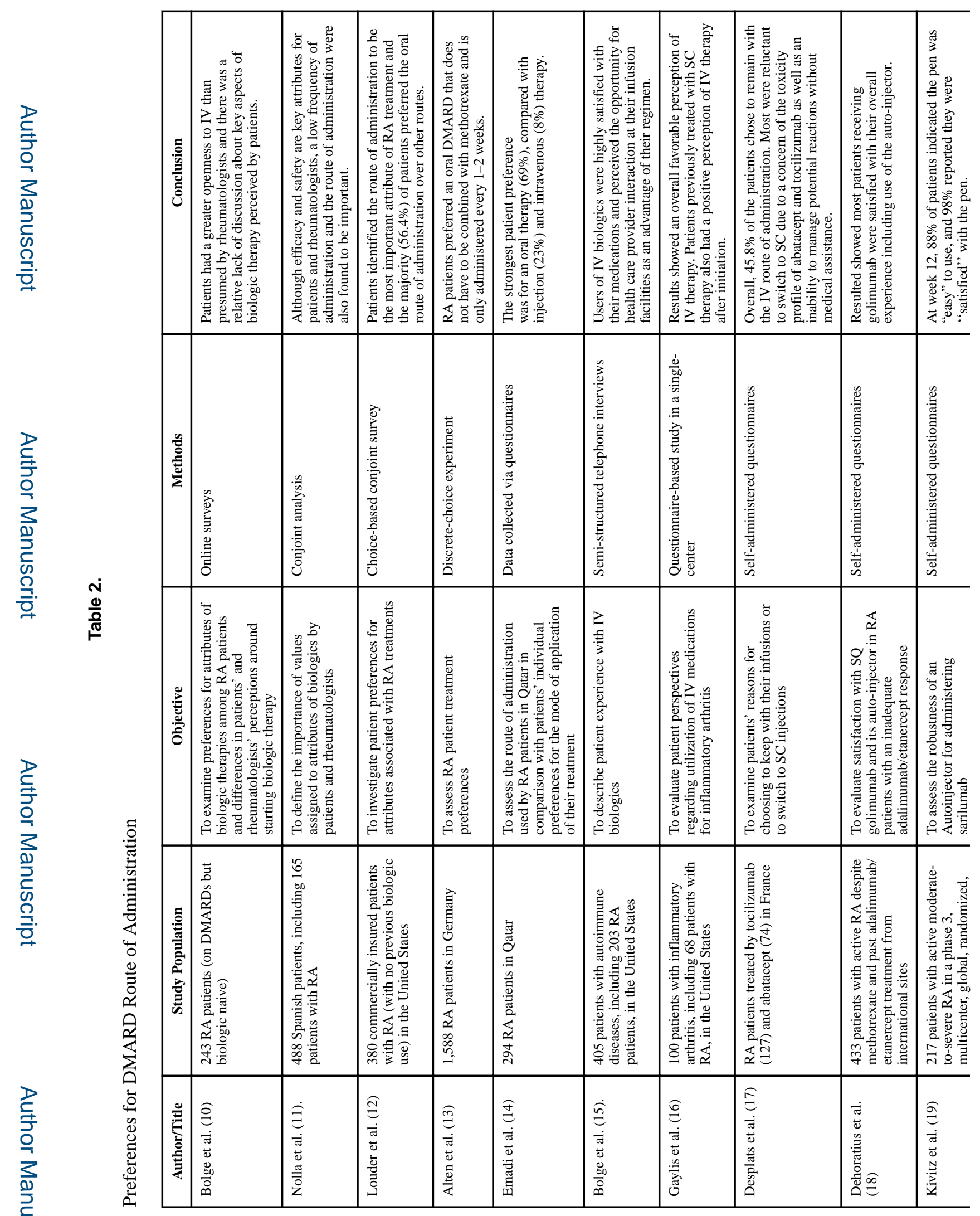

Curr Opin Rheumatol. Author manuscript; available in PMC 2020 May 01. 


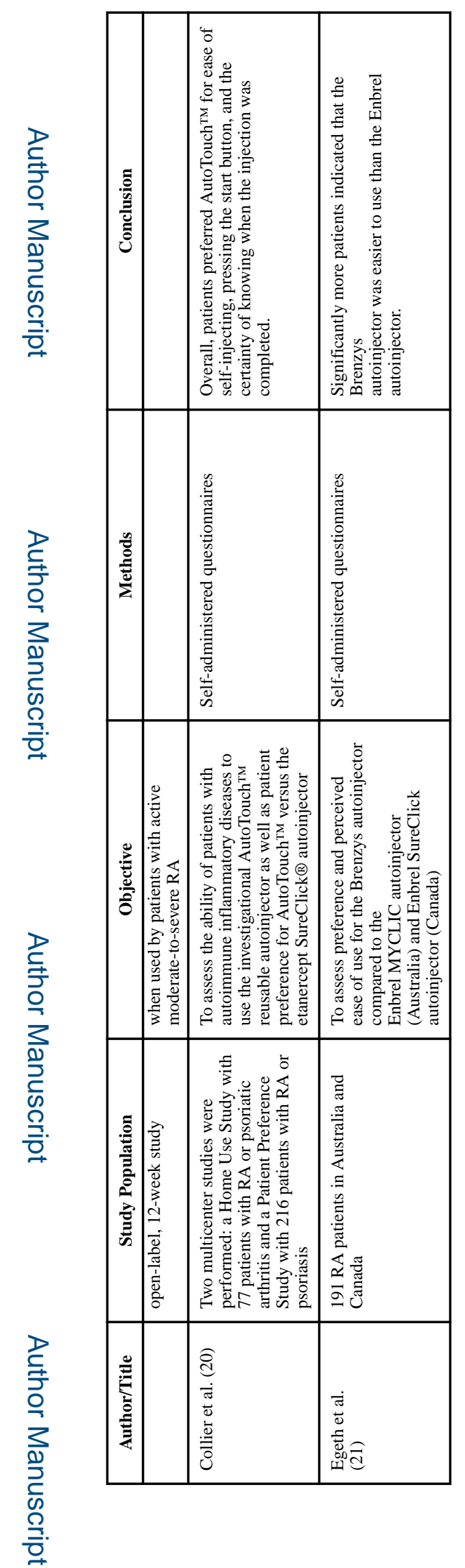

Curr Opin Rheumatol. Author manuscript; available in PMC 2020 May 01. 